\title{
ON THE BACKWARD EXTENSION OF POSITIVE DEFINITE HAMBURGER MOMENT SEQUENCES 1
}

FRED M. WRIGHT

1. Introduction. Throughout this paper, let

$$
\left\{\mu_{n}\right\} \quad(n=0,1,2, \cdots)
$$

be a given positive definite Hamburger moment sequence, that is, (1.1) is a Hamburger moment sequence such that

$$
\Delta_{0, n}>0 \quad(n=0,1,2, \cdots),
$$

where

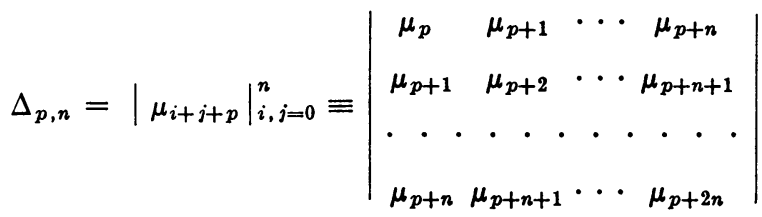

$$
\begin{aligned}
& (p, n=0,1,2, \cdots) \text {. }
\end{aligned}
$$

The condensed determinant notation indicated in (1.3) will be used whenever possible in order to save space. Let

$$
\frac{a_{0}^{2}}{z+b_{1}}-\frac{a_{1}^{2}}{z+b_{2}}-\frac{a_{2}^{2}}{z+b_{3}}-\cdots
$$

be the $J$-fraction expansion of the formal power series $\sum_{n=0}^{\infty} \mu_{n} / z^{n+1}$, and let

$$
\begin{aligned}
& X_{p}(z)=\frac{A_{p}(z)}{a_{0} a_{1} \cdots a_{p}} \\
& Y_{p}(z)=\frac{B_{p}(z)}{a_{0} a_{1} \cdots a_{p}}
\end{aligned} \quad(p=0,1,2, \cdots),
$$

where $A_{p}(z)$ and $B_{p}(z)$ denote the $p$ th numerator and denominator, respectively, of (1.4). It is readily shown that the sequences

Presented to the Society, September 3, 1954 ; received by the editors December 27, 1954 and, in revised form, June 10, 1955.

1 Some of the ideas in this paper are contained in a thesis submitted to Northwestern University and prepared under the direction of Professor Walter T. Scott. 


$$
\left\{\mu_{n}^{(i)}\right\} \equiv\left\{\mu_{n+2 i}\right\} \quad(i=1,2,3, \cdots ; n=0,1,2, \cdots)
$$

are positive definite Hamburger moment sequences. By a first backward extension of (1.1) we shall mean a positive definite Hamburger moment sequence

$$
\left\{\lambda_{0}, \lambda_{1}, \lambda_{2}\left(=\mu_{0}\right), \lambda_{3}\left(=\mu_{1}\right), \cdots\right\},
$$

by a second backward extension of (1.1) we shall mean a first backward extension of (1.6), etc.

In this paper, a relatively simple discussion of the problem of extending (1.1) backward is given which is based essentially only on the properties of the polynomials (1.5) given in the following three lemmas.

Lemma 1.1. If the two series

$$
\sum_{n=0}^{\infty}\left|X_{n}(z)\right|^{2}, \quad \sum_{n=0}^{\infty}\left|Y_{n}(z)\right|^{2}
$$

both converge for a single value $z=z_{0}$, then these series converge for all $z$, and the convergence is uniform throughout each closed circular region $|z| \leqq M$.

Lemma 1.2. (1.1) is an indeterminate Hamburger moment sequence if and only if the series (1.7) both converge for $z=0$.

Lemma 1.3. If the series $\sum_{n=0}^{\infty}\left|Y_{n}(z)\right|^{2}$ converges for a value $z=z_{0}$ such that $\operatorname{Im} z_{0}>0$, then the series $\sum_{n=0}^{\infty}\left|X_{n}(z)\right|^{2}$ also converges for $z=z_{0}$.

In $\S 2$ a necessary and sufficient condition for (1.1) to be extendable backward once is obtained which is first stated in terms of the polynomials (1.5) and then in terms of the given moments (1.1). It is interesting to note that this condition, as given in Theorem 2.1, contains as a special case the principal result due to H. S. Wall [1 $]^{2}$ and proved again by the author [2] relative to the first backward extension of positive definite Stieltjes moment sequences. In $\$ 3$ we obtain further results relative to the first backward extension of (1.1), and we then apply these results to the problem of the second backward extension of (1.1). It is to be noted that Hamburger [3] proved some of the results given in $\S 3$ by the use of properties of distribution functions and certain quadratic forms in deriving a well-known necessary and sufficient condition involving the moments themselves for (1.1) to be an indeterminate Hamburger moment sequence; this con-

\footnotetext{
2 Numbers in brackets refer to references at the end of this paper.
} 
dition, which was proved again by M. Riesz [4], is proved quite simply in $\$ 4$ by the use of results of $\$ 2$ and $\$ 3$. H. S. Wall [5] has also proved some of the results of $\S 3$ by the use of properties of integrals and results of Hamburger.

2. A necessary and sufficient condition for (1.1) to be extendable backward once. Using the definitions of the polynomials (1.5), we have, after some computation, the formulas

$(2.1)^{\prime} \quad F_{n}\left(z ; z_{0}\right) \equiv \sum_{p=0}^{n} Y_{p}(z) Y_{p}\left(z_{0}\right)=-\frac{1}{\Delta_{0, n}} \cdot\left|\begin{array}{cc}0 & z_{0}^{j} \\ z^{i} & \mu_{i+j}\end{array}\right|_{i, j=0}^{n}$

$(n=0,1,2, \cdots)$,

$(2.1)^{\prime \prime} \quad G_{n}\left(z ; z_{0}\right) \equiv \sum_{p=0}^{n} Y_{p}(z) X_{p}\left(z_{0}\right)=-\frac{1}{\Delta_{0, n}} \cdot\left|\begin{array}{cc}0 & \sum_{k=0}^{j-1} \mu_{j-1-k} z_{0}^{k} \\ z^{i} & \mu_{i+j}\end{array}\right|_{i, j=0}^{n}$

$$
(n=1,2,3, \cdots)
$$

and

$$
\begin{aligned}
H_{n}\left(z ; z_{0}\right) & \equiv \sum_{p=0}^{n} X_{p}(z) X_{p}\left(z_{0}\right) \\
& =-\frac{1}{\Delta_{0, n}} \cdot \mid \begin{array}{cc}
0 & \sum_{k=0}^{j-1} \mu_{j-1-k} z_{0}^{k} \\
\sum_{k=0}^{i-1} \mu_{i-1-k} z^{k} & \left.\mu_{i+j}\right|_{i, j=0} ^{n} \\
(n=1,2,3, \cdots),
\end{array}
\end{aligned}
$$

where we shall understand that $\sum_{k=0}^{-1} \mu_{-1-k} z^{k} \equiv 0$. In order to save space in the printing of certain formulas, we shall henceforth denote the quantities $F_{n}(0 ; 0), G_{n}(0 ; 0)$ and $H_{n}(0 ; 0)$ by the symbols $F_{n}$, $G_{n}$ and $H_{n}$, respectively.

If $\mu_{-2}$ and $\mu_{-1}$ are any two real numbers, then we have from (2.1)', $(2.1)^{\prime \prime}$ and $(2.1)^{\prime \prime \prime}$ that

$$
\begin{array}{r}
\left|\mu_{i+j-2}\right|_{i, j=0}^{n+2}=\Delta_{0, n+1} \cdot\left\{\mu_{-2}-\sum_{p=0}^{n+1}\left[X_{p}(0)+\mu_{-1} Y_{p}(0)\right]^{2}\right\} \\
(n=0,1,2, \cdots) .
\end{array}
$$

Thus we have 
THEOREM 2.1. (1.1) can be extended backward once if and only if the intersection

$$
\begin{aligned}
R & \equiv\left\{(x, y) ; \sum_{p=0}^{\infty}\left[X_{p}(0)+y Y_{p}(0)\right]^{2}<+\infty \cdot x\right. \\
& \left.\geqq \sum_{p=0}^{\infty}\left[X_{p}(0)+y Y_{p}(0)\right]^{2}\right\}
\end{aligned}
$$

of the open and nested parabolic regions

$$
\begin{aligned}
R_{n} & \equiv\left\{(x, y) ; x>\sum_{p=0}^{n}\left[X_{p}(0)+y Y_{p}(0)\right]^{2}\right\} \\
& =\left\{(x, y) ;\left(x-x_{n}\right)>F_{n} \cdot\left(y-y_{n}\right)^{2}\right\} \quad(n=0,1,2,3, \cdots)
\end{aligned}
$$

is not vacuous, where

$$
x_{n}=H_{n}-\left(G_{n}\right)^{2} / F_{n} \quad(n=0,1,2, \cdots)
$$

and

$$
y_{n}=-G_{n} / F_{n}, \quad(n=0,1,2, \cdots) .
$$

Moreover, in this case (1.6) is a positive definite Hamburger moment sequence if and only if $\left(\lambda_{0}, \lambda_{1}\right) \in R$.

From Lemma 1.2 and Theorem 2.1 we have

CoRollary 2.1. An indeterminate Hamburger moment sequence (1.1) can always be extended backward once, and the intersection $R$ of the parabolic regions (2.4) is given by

$$
R=\left\{(x, y) ;\left(x-x^{(0)}\right) \geqq \sum_{p=0}^{\infty}\left[Y_{p}(0)\right]^{2} \cdot\left(y-y^{(0)}\right)^{2}\right\},
$$

where

$$
\lim _{n \rightarrow \infty} x_{n}=x^{(0)}
$$

and

$$
\lim _{n \rightarrow \infty} y_{n}=y^{(0)}
$$

We now prove

LEMмA 2.1. If (1.1) is a determinate Hamburger moment sequence such that $\sum_{p=0}^{\infty}\left[Y_{p}(0)\right]^{2}<+\infty$, then the non-negative monotone nondecreasing quantities (2.5) have the limit $+\infty$. 
Proof. Suppose that the limit $x^{(0)}$ in $(2.8)$ is finite. Then, since $\sum_{p=0}^{\infty}\left[X_{p}(0)\right]^{2}=+\infty$ in view of Lemma 1.2 and our hypothesis that (1.1) is a determinate Hamburger moment sequence such that $\sum_{p=0}^{\infty}\left[Y_{p}(0)\right]^{2}<+\infty$, it follows that the quantities

$$
\left(G_{n}\right)^{2} / F_{n}, \quad(n=0,1,2, \cdots),
$$

are unbounded, so that the quantities $G_{n}(n=0,1,2, \cdots)$, and hence also the quantities (2.6), are unbounded. However, since $\left(x_{n}, y_{n}\right) \in \bar{R}_{0},(n=0,1,2, \cdots)$, where

$$
\bar{R}_{0} \equiv\left\{(x, y) ; x \geqq y^{2}\right\},
$$

it follows that

$$
-\left(x^{(0)}\right)^{1 / 2} \leqq y_{n} \leqq\left(x^{(0)}\right)^{1 / 2} \quad(n=0,1,2, \cdots) .
$$

Thus, the supposition that $x^{(0)}$ is finite leads to a contradiction.

This completes the proof of Lemma 2.1.

From Theorem 2.1 and Lemma 2.1 we have

CoRollary 2.2. A determinate Hamburger moment sequence (1.1) such that $\sum_{p=0}^{\infty}\left[Y_{p}(0)\right]^{2}<+\infty$ cannot be extended backward once.

We now prove

LEMma 2.2. If (1.1) is a determinate Hamburger moment sequence such that the non-negative monotone nondecreasing quantities (2.5) have a finite limit $x^{(0)}$, then the quantities (2.6) have a finite limit.

Proof. From Lemma 2.1 it follows that $\sum_{p=0}^{\infty}\left[Y_{p}(0)\right]^{2}=+\infty$. If

$$
\begin{aligned}
\bar{R}_{n} & \equiv\left\{(x, y) ; x \geqq \sum_{p=0}^{n}\left[X_{p}(0)+y Y_{p}(0)\right]^{2}\right\} \\
& =\left\{(x, y) ;\left(x-x_{n}\right) \geqq F_{n} \cdot\left(y-y_{n}\right)^{2}\right\} \quad(n=0,1,2, \cdots),
\end{aligned}
$$

then

$$
\left(x_{n+j}, y_{n+j}\right) \in \bar{R}_{n} \quad(n, j=0,1,2, \cdots) .
$$

Thus

$$
\left|y_{n+j}-y_{n}\right| \leqq\left\{\left(x^{(0)}-x_{n}\right) / F_{n}\right\}^{1 / 2} \quad(n, j=0,1,2, \cdots) .
$$

If $\epsilon$ is any given positive number, let $N_{\epsilon}$ be a positive integer such that

$$
n>N_{\epsilon} \cdot \supset \cdot F_{n}>1 / \epsilon \cdot\left(x^{(0)}-x_{n}\right)<\epsilon,
$$

so that

$$
n>N_{\epsilon} \cdot j=0,1,2, \cdots \cdot \supset \cdot\left|y_{n+j}-y_{n}\right|<\epsilon .
$$


Therefore, the quantities (2.6) have a finite limit by the Cauchy criterion for the convergence of a sequence.

This completes the proof of Lemma 2.2.

From Lemma 1.2, Theorem 2.1 and Lemma 2.2 we have

TheOREM 2.2. (1.1) can be extended backward once if and only if the non-negative monotone nondecreasing quantities (2.5) have a finite limit $x^{(0)}$. In this case, the quantities (2.6) also have a finite limit $y^{(0)}$.

From Theorem 2.1, Lemma 2.1 and Theorem 2.2 we have

CoROLlaRY 2.3. If (1.1) is a determinate Hamburger moment sequence which can be extended backward once, then the intersection $R$ of the parabolic regions (2.4) is given by

$$
R=\left\{(x, y) ; x \geqq x^{(0)} \cdot y=y^{(0)}\right\} .
$$

Using the theory of Laplace minors, we can easily show that if $\mu_{-1}$ is any real number then

$$
\begin{aligned}
\left|\begin{array}{cc}
0 & \mu_{j-1} \\
\mu_{i-1} & \mu_{i+j}
\end{array}\right|_{i, j=0}^{n} \cdot \Delta_{2, n-1}=\Delta_{0, n} & \cdot\left|\begin{array}{cc}
0 & \mu_{j-1} \\
\mu_{i-1} & \mu_{i+j}
\end{array}\right|_{i, j=1}^{n} \\
& -\left(\left|\begin{array}{cc}
\mu_{-1} & \mu_{j-1} \\
\mu_{i} & \mu_{i+j}
\end{array}\right|_{i, j=1}^{n}\right)^{2}(n=1,2, \cdots) .
\end{aligned}
$$

Thus, in view of $(2.1)^{\prime},(2.1)^{\prime \prime}$, and $(2.1)^{\prime \prime \prime}$ as well as Theorem 2.2 we have

THEOREM 2.3. (1.1) can be extended backward once if and only if the non-negative monotone nondecreasing determinant ratios

$$
\frac{-1}{\Delta_{2, n-1}} \cdot\left|\begin{array}{cc}
0 & \mu_{j-1} \\
\mu_{i-1} & \mu_{i+j}
\end{array}\right|_{i, j=1} \quad(n=1,2,3, \cdots)
$$

have a finite limit.

3. Some further results. If $\mu_{-2}$ and $\mu_{-1}$ are any two real numbers, then from $(2.1)^{\prime},(2.1)^{\prime \prime}$ and $(2.1)^{\prime \prime \prime}$ we have that

$$
\begin{array}{r}
\left|\begin{array}{cc}
0 & \mu_{j-2} \\
\mu_{i-2} & \mu_{i+j}
\end{array}\right|_{i, j=0}^{n+1} \cdot \frac{-1}{\Delta_{0, n+1}}=\sum_{p=0}^{n+1}\left[X_{p}^{\prime}(0)+\mu_{-2} Y_{p}(0)+\mu_{-1} Y_{p}^{\prime}(0)\right]^{2} \\
(n=1,2,3, \cdots) .
\end{array}
$$

We now prove

Lemma 3.1. Suppose that (1.1) is extendable backward once and that (1.6) is a first backward extension of (1.1). Let 


$$
\frac{c_{0}^{2}}{z+d_{1}}-\frac{c_{1}^{2}}{z+d_{2}}-\frac{c_{2}^{2}}{z+d_{3}}-\cdots
$$

be the $J$-fraction expansion of the formal power series $\sum_{n=0}^{\infty} \lambda_{n} / z^{n+1}$, and let

$$
\begin{aligned}
& V_{p}(z)=\frac{C_{p}(z)}{c_{0} c_{1} \cdots c_{p}} \\
& W_{p}(z)=\frac{D_{p}(z)}{c_{0} c_{1} \cdots c_{p}}
\end{aligned} \quad(p=0,1,2, \cdots),
$$

where $C_{p}(z)$ and $D_{p}(z)$ denote the pth numerator and denominator, respectively, of (3.2). Then,

$$
\begin{aligned}
\sum_{p=0}^{n+1}\left[W_{p}(0)\right]^{2}=\left\{\lambda_{0}-\sum_{p=0}^{n}\left[X_{p}(0)+\lambda_{1} Y_{p}(0)\right]^{2}\right\}^{-1} & (n=1,2,3, \cdots),
\end{aligned}
$$

and for arbitrary complex $z$ we have that

$$
\begin{aligned}
\sum_{p=0}^{n+1}\left|W_{p}(z)\right|^{2}= & |z|^{2} \cdot \sum_{p=0}^{n}\left|Y_{p}(z)\right|^{2}+\mid z \cdot\left[G_{n}(z ; 0)+\lambda_{1} F_{n}(z ; 0)\right] \\
& -\left.1\right|^{2} \cdot \sum_{p=0}^{n+1}\left[W_{p}(0)\right]^{2} \quad(n=1,2,3, \cdots) .
\end{aligned}
$$

Proof. (3.4) follows from (2.1)' and (2.2). (3.5) follows from (2.1) and $(2.1)^{\prime \prime}$, as well as the determinant formula

$$
\begin{aligned}
&\left|\begin{array}{cc}
0 & \bar{z}^{j} \\
z^{i} & \mu_{i+j-2}
\end{array}\right|_{i, j=0}^{n+1} \cdot \Delta_{0, n}=\left|\mu_{i+j-2}\right|_{i, j=0}^{n+1} \cdot\left|\begin{array}{cc}
0 & \bar{z}^{i} \\
z^{i} & \mu_{i+j-2}
\end{array}\right|_{i, j=1}^{n+1} \\
&-\left|\begin{array}{cc}
1 & \mu_{j-2} \\
z^{i} & \mu_{i+j-2}
\end{array}\right|_{i, j=1}^{n+1} \\
& \qquad \cdot\left|\begin{array}{cc}
1 & \bar{z}^{j} \\
\mu_{i-2} & \mu_{i+j-2}
\end{array}\right|_{i, j=1}^{n+1} \quad(n=1,2,3, \cdots)
\end{aligned}
$$

which holds for arbitrary real numbers $\mu_{-2}$ and $\mu_{-1}$.

This completes the proof of Lemma 3.1.

We now prove the following theorem.

THEOREM 3.1. If (1.1) is a determinate Hamburger moment sequence which is extendable backward once, and if (1.6) is a first backward extension of (1.1), then (1.6) is a determinate Hamburger moment sequence. Moreover, (1.6) can be extended backward once if and only if $\lambda_{0}=x^{(0)}$ and 


$$
\sum_{p=0}^{\infty}\left[X_{p}^{\prime}(0)+x^{(0)} Y_{p}(0)+y^{(0)} Y_{p}^{\prime}(0)\right]^{2}<+\infty,
$$

where $x^{(0)}$ and $y^{(0)}$ are given by (2.8) and (2.9), respectively.

Proof. Let (3.2) and (3.3) be as in Lemma 3.1. From Lemma 1.1, Lemma 1.2 and Lemma 1.3 it follows that $\sum_{p=0}^{\infty}\left|Y_{p}(z)\right|^{2}=\infty$ for arbitrary complex $z$ such that $\operatorname{Im} z>0$, and hence from (3.5) we have that $\sum_{p=0}^{\infty}\left|W_{p}(z)\right|^{2}=\infty$ for arbitrary complex $z$ such that $\operatorname{Im} z>0$; therefore, (1.6) is a determinate Hamburger moment sequence in view of Lemma 1.1 and Lemma 1.2. From Theorem 2.1, Corollary 2.3 and (3.4) it follows that $\sum_{p=0}^{\infty}\left[W_{p}(0)\right]^{2}=\infty$ if and only if $\lambda_{0}=x^{(0)}$; therefore, from Theorem 2.1, Corollary 2.2, Corollary 2.3, Theorem 2.3 and (3.1) we have that (1.6) can be extended backward once if and only if $\lambda_{0}=x^{(0)}$ and (3.6) holds.

This completes the proof of Theorem 3.1.

We next prove

THEOREM 3.2. If (1.1) is an indeterminate Hamburger moment sequence, and if (1.6) is a first backward extension of (1.1), then (1.6) is an indeterminate Hamburger moment sequence if and only if $\left(\lambda_{0}, \lambda_{1}\right)$ is an interior point of the closed parabolic region $R$ in (2.7). Moreover, (1.6) can always be extended backward once.

Proof. Again, let (3.2) and (3.3) be as in Lemma 3.1. From Lemma 1.1 and Lemma 1.2 it follows that $\sum_{p=0}^{\infty}\left|Y_{p}(z)\right|^{2}<+\infty$ and $\lim _{n \rightarrow \infty}\left|z \cdot\left[G_{n}(z ; 0)+\lambda_{1} F_{n}(z ; 0)\right]-1\right|^{2}$ exists and is finite for every complex $z$, so that the series $\sum_{p=0}^{\infty}\left|W_{p}(z)\right|^{2}$ converges for every complex $z$ if and only if $\sum_{p=0}^{\infty}\left[W_{p}(0)\right]^{2}<+\infty$ in view of (3.5); therefore, since $\sum_{p=0}^{\infty}\left[W_{p}(0)\right]^{2}<+\infty$ if and only if $\left(\lambda_{0}, \lambda_{1}\right)$ is an interior point of the closed parabolic region $R$ of (2.7) in view of Theorem 2.1, Corollary 2.1 and (3.4), we have that (1.6) is an indeterminate Hamburger moment sequence if and only if $\left(\lambda_{0}, \lambda_{1}\right)$ is an interior point of the closed parabolic region $R$ of (2.7) in view of Lemma 1.1, Lemma 1.2 and Lemma 1.3. From Lemma 1.1 and Lemma 1.2 it follows that the series $\sum_{p=0}^{\infty}\left[X_{p}^{\prime}(0)+x Y_{p}(0)+y Y_{p}^{\prime}(0)\right]^{2}$ converges for arbitrary real $x$ and $y$; therefore, (1.6) is extendable backward once in view of Theorem 2.3 and (3.1).

This completes the proof of Theorem 3.2.

4. A new proof of a known necessary and sufficient condition for (1.1) to be an indeterminate Hamburger moment sequence. We now show how results of $\$ 2$ and $\$ 3$ can be used to give a new and relatively simple proof of 
THEOREM 4.1. (1.1) is an indeterminate Hamburger moment sequence if and only if the positive nonincreasing determinant ratios

$$
\Delta_{0, n} / \Delta_{4, n-2} \quad(n=2,3,4 \cdots)
$$

have a positive limit.

Proof. Let

$$
\frac{\left[a_{0}^{(1)}\right]^{2}}{z+b_{1}^{(1)}}-\frac{a\left[{ }_{1}^{(1)}\right]^{2}}{z+b_{2}^{(1)}}-\frac{\left[a_{2}^{(1)}\right]^{2}}{z+b_{8}^{(1)}}-\cdots
$$

be the $J$-fraction expansion of the formal power series $\sum_{n=0}^{\infty} \mu_{n}^{(1)} / z^{n+1}$, where

$$
\left\{\mu_{n}^{(1)}\right\} \equiv\left\{\mu_{n+2}\right\} \quad(n=0,1,2, \cdots)
$$

and let

$$
\begin{aligned}
& X_{p}^{(1)}(z)=\frac{A_{p}^{(1)}(z)}{a_{0}^{(1)} a_{1}^{(1)} \cdots a_{p}^{(1)}} \quad(p=0,1,2, \cdots), \\
& Y_{p}^{(1)}(z)=\frac{B_{p}^{(1)}(z)}{a_{0}^{(1)} a_{1}^{(1)} \cdots a_{p}^{(1)}}
\end{aligned}
$$

where $A_{p}^{(1)}(z)$ and $B_{p}^{(1)}(z)$ denote the $p$ th numerator and denominator, respectively, of (4.2). From $(2.1)^{\prime}$ it follows that

$$
\sum_{p=0}^{n}\left[Y_{p}(0)\right]^{2}=\Delta_{2, n-1} / \Delta_{0, n} \quad(n=1,2,3, \cdots)
$$

and

so that

$$
\sum_{p=0}^{n-1}\left[Y_{p}^{(1)}(0)\right]^{2}=\Delta_{4, n-2} / \Delta_{2, n-1} \quad(n=2,3,4, \cdots)
$$

$$
\begin{aligned}
\Delta_{0, n} / \Delta_{4, n-2}=\left\{\sum_{p=0}^{n}\left[Y_{p}(0)\right]^{2} \cdot \sum_{p=0}^{n-1}\left[Y_{p}^{(1)}(0)\right]^{2}\right\}^{-1} & \\
& (n=2,3,4, \cdots) .
\end{aligned}
$$

If (1.1) is an indeterminate Hamburger moment sequence, then (4.3) must also be an indeterminate Hamburger moment sequence in view of Theorem 3.1; therefore, $\sum_{p=0}^{\infty}\left[Y_{p}(0)\right]^{2}<+\infty$ and $\sum_{p=0}^{\infty}\left[Y_{p}^{(1)}(0)\right]^{2}<+\infty$ in view of Lemma 1.2. Thus, if (1.1) is an 
indeterminate Hamburger moment sequence, the ratios (4.1) have a positive limit in view of (4.5).

If the ratios (4.1) have a positive limit, then $\sum_{p=0}^{\infty}\left[Y_{p}(0)\right]^{2}<+\infty$ and $\sum_{p=0}^{\infty}\left[Y_{p}^{(1)}(0)\right]^{2}<+\infty$ in view of (4.5). Thus, if the ratios (4.1) have a positive limit, it follows that (4.3) must be an indeterminate Hamburger moment sequence in view of Corollary 2.2 and the convergence of the series $\sum_{p=0}^{\infty}\left[Y_{p}^{(1)}(0)\right]^{2}$; it then follows from Theorem 3.2 , Corollary 2.2 and the convergence of the series $\sum_{p=0}^{\infty}\left[Y_{p}(0)\right]^{2}$ that (1.1) is an indeterminate Hamburger moment sequence.

This completes the proof of Theorem 4.1.

\section{REFERENCES}

1. H. S. Wall, On the Pade approximants associated with the continued fraction and series of Stieltjes, Trans. Amer. Math. Soc. vol. 31 (1929) pp. 91-116.

2. F. M. Wright, A transformation for S-fractions, Proc. Amer. Math. Soc. vol. 5 (1954) pp. 888-901.

3. H. Hamburger, Über eine Erweiterung des Stieltjesschen Momentproblems. Teil III, Math. Ann. vol. 82 (1921) pp. 168-187.

4. M. Riesz, Sur le problème des moments. Première note, Arkiv för Mathematik, Astronomi och Fysik vol. 16 (1921) article 12.

5. H. S. Wall, On the Pade approximants associated with a positive definite power series, Trans. Amer. Math. Soc. vol. 33 (1931) pp. 511-532.

Iowa State College and

NORTHWESTERN UNIVERSITY 